\title{
Spatial variation of lithospheric heterogeneity on the globe as revealed from transverse amplitudes of short-period teleseismic $P$-waves
}

\author{
Mungiya Kubanza, Takeshi Nishimura, and Haruo Sato \\ Department of Geophysics, Graduate School of Science, Tohoku University, Aramaki-aza Aoba 6-3, Aoba-ku, Sendai 980-8578, Japan
}

(Received March 29, 2006; Revised August 12, 2006; Accepted August 24, 2006; Online published October 13, 2006)

\begin{abstract}
We systematically characterized the medium heterogeneity of the lithosphere by analyzing transversecomponent amplitudes of teleseismic $P$-waves from shallow earthquakes in short periods of 0.5 to $4 \mathrm{~Hz}$. Normalized transverse amplitudes, which are less affected by a layered structure, were measured at each station of the IRIS GSN network. The data revealed significant regional differences in lateral heterogeneity of the lithosphere, with small amplitudes observed at stations on stable continents and large normalized transverse amplitudes observed in seismically active regions, such as island arcs or collision zones. These spatial changes are consistent with the tectonic settings of each station. However, large normalized transverse amplitudes were also observed in regions of very low seismicity and in regions where no seismic activity has been recognized, which may indicate the existence of medium heterogeneity in the lithosphere that has been formed in ancient times.
\end{abstract}

Key words: Heterogeneity, lithosphere, transverse amplitude, teleseismic $P$-wave, seismicity, tectonics.

\section{Introduction}

Heterogeneity of the earth's lithosphere is considered to be mainly related with the mechanism by which the earth's crust is formed, volcanic processes, and the nature of the active seismic zones. As such, the heterogeneous structure of the lithosphere has been extensively studied using seismic waves generated by earthquakes and by man-made sources. For example, tomography techniques have succeeded in clarifying the large-scale heterogeneous structure of the earth (e.g., Megnin and Romanowicz, 2000). Analyses of seismic coda waves have also been used to evaluate small-scale heterogeneity (e.g., Fehler et al., 1992). These analyses have resulted in detailed views of seismic structure and an accumulation of valuable data on the dynamics and evolution of the earth. Regions with a dense network of stations and high seismicity are particularly valuable for providing data on spatial changes in seismic velocity, the fluctuation of elastic parameters, and attenuation factors.

Teleseismic $P$-waves, which are also recorded at stations where seismicity is low, have been analyzed during systematic evaluations of the lithosphere of the globe. For example, receiver function analyses have been applied in many regions, revealing a detailed view of the layered structure in the upper mantle and crust by analyzing simultaneously vertical and radial components of teleseismic $P$-waves (e.g., Langston, 1979). However, to date, few investigations have been carried out on the lateral variations of heterogeneity, which are not detected by receiver function analysis. Envelope modeling of teleseismic $P$-waves was first performed using the energy-flux model developed by Korn (1993) for the study of lithospheric inhomogeneity. Nishimura et al.

Copy right(c) The Society of Geomagnetism and Earth, Planetary and Space Sciences (SGEPSS); The Seismological Society of Japan; The Volcanological Society of Japan; The Geodetic Society of Japan; The Japanese Society for Planetary Sciences; TERRAPUB.
(2002) first analyzed the transverse amplitude of teleseismic $P$-waves to evaluate lateral heterogeneity in the lithosphere, ultimately showing that strong heterogeneity is recognizable in and around the tectonically active regions and estimating the scattering strength in depth assuming a single scattering process. However, their study was restricted to the western Pacific region due to the limited hypocentral distribution of deep earthquakes as they needed a simple source time function for evaluating the depth dependence of the scattering properties.

In the study reported here, therefore, we focused on analyzing short-period teleseismic $P$-coda waves from shallow earthquakes, which occur more widely around the world, with the aim of characterizing the medium heterogeneity of the globe. Sato (2006) recently showed that the ratio of the peak intensity of the transverse component to the sum of the three components of the traces is proportional to the fluctuation, correlation length, and thickness of heterogeneity, based on the Markov approximation for a random medium characterized by Gaussian autocorrelation function. Hence, we also discuss the spatial variation of the partition of $P$ wave energy into its transverse component in relation to tectonic settings in order to classify the lithospheric heterogeneities. We also show that the normalized transverse amplitude of teleseismic $P$-waves recorded at large distances can be a useful parameter by which to represent medium heterogeneity-mainly beneath the receivers and not close to the focal zone of the shallow earthquakes.

\section{Measurement of Transverse Amplitudes}

We selected waveform data from the broadband threecomponent traces stored in the IRIS FARM archives that covered the period from January 1998 through to December 2002. We used shallow earthquake records with a focal depth of less than $35 \mathrm{~km}$ and a body wave magnitude 


\section{$1-2 \mathrm{~Hz}$}

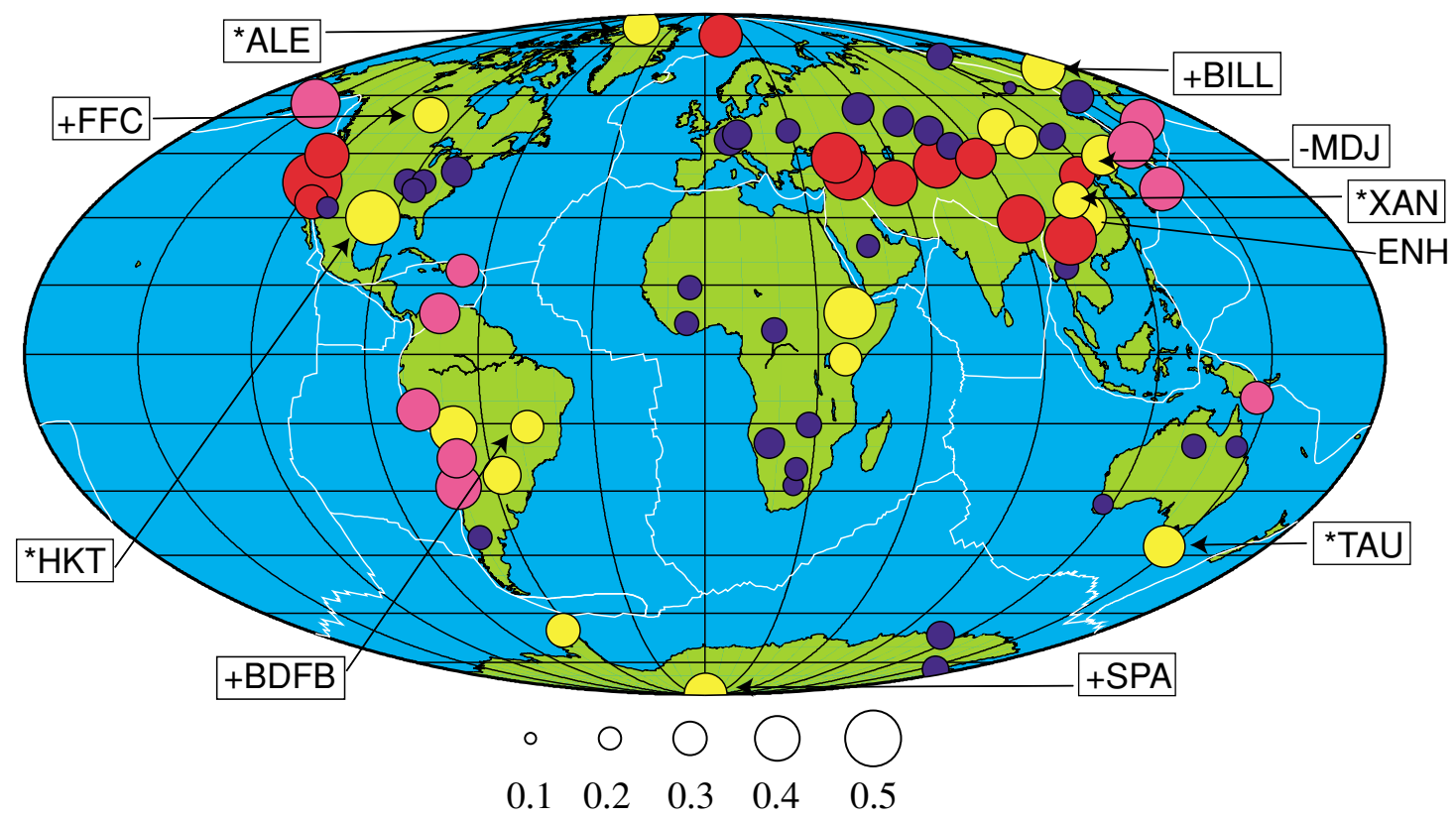

Fig. 1. Spatial variation of normalized transverse amplitudes $\langle A\rangle$ at frequency band of $1-2 \mathrm{~Hz}$. The radius of each circle is proportional to $\langle A\rangle$ and the color scale represents the category shown in Fig. 2: Blue (A), yellow (B), red (C), and pink (D). Arrows indicate the stations where no earthquake of $\mathrm{M} \geq 5$ has been observed. Stars $(*)$ preceding the station code indicate stations where smaller earthquakes $(\mathrm{M} \geq 3)$ have been detected. Plus $(+)$ signs indicate stations where no seismic and volcanic activities have been found. Minus $(-)$ signs indicate a station located very close to a Holocene volcano (Jingpohu).

of between 5 and 6 . The source-receiver range was restricted to 30-60 degrees to avoid contamination from core reflected phases and converted phases from the mantle transition zone. Since seismic wave propagation and scattering through the randomly heterogeneous structure of the earth indicate frequency dependence (Sato and Fehler, 1998), seismograms were bandpass filtered for the ranges of $0.5-1$ $\mathrm{Hz}, 1-2 \mathrm{~Hz}$, and 2-4 Hz where large signal-to-noise ratios are observed. Among the 13,510 velocity traces collected, a set of 3378 traces with a signal-to-noise ratio greater than 10 at 72 stations was used for the following analysis.

We defined the normalized transverse amplitude of teleseismic $P$-waves as follows. (1) For each event-station pair, we extracted three-component velocity seismograms of the teleseismic $P$-waves for the first $20 \mathrm{~s}$ following their arrivals. (2) We calculated the total seismic energy of the three-component seismograms, $E^{\text {total }}=E^{\text {ud }}+E^{\text {rd }}+E^{\text {tr }}$, where $E^{\text {ud }}, E^{\text {rd }}$, and $E^{\text {tr }}$ are the seismic energy in the vertical, radial, and transverse components, respectively. The seismic energy was calculated by multiplying the density and squared velocity of each component for $20 \mathrm{~s}$. (3) We calculated the energy partition of the $P$-coda waves into the transverse component and took its root-mean-square amplitude, which represents the normalized transverse amplitude; this amplitude is expressed as $A=\sqrt{E^{\text {tr }} / E^{\text {total }}}$. (4) We further took the average $\langle A\rangle$ of all the normalized transverse amplitudes over events at each station. Hereafter, we call $\langle A\rangle$ simply the "normalized transverse amplitude". These amplitudes were calculated for the frequency bands of $0.5-$ $1 \mathrm{~Hz}, 1-2 \mathrm{~Hz}$, and 2-4 Hz.

The observed normalized transverse amplitudes ranged from 0.08 to 0.43 at $0.5-1 \mathrm{~Hz}, 0.11$ to 0.53 at $1-2 \mathrm{~Hz}$, and 0.16 to 0.53 at $2-4 \mathrm{~Hz}$. The averages for all of the stations at these three frequency bands were estimated to be $0.22,0.29$ and 0.31 , respectively. In the followings, we only show the results of the 1-2 $\mathrm{Hz}$ band because overall characteristics were found to be the same in these three frequency bands.

\section{Spatial Distribution of Transverse Amplitudes}

The geographical distribution of the observed normalized transverse amplitudes is shown in Fig. 1. The radius of the circle plotted at each station represents the normalized transverse amplitude according to the scale shown at the bottom of the figure. The color scale of each circle represents the classification which will be given in Fig. 2. The observed normalized transverse amplitudes indicate significant regional differences in lithospheric heterogeneity. In the Eurasian continent and western Pacific, most of large normalized transverse amplitudes are observed at stations located on tectonically active regions, such as along the Arabia-Eurasia plate boundary, the collision zone of the Indian and Eurasian plates, and along the island arcs in the western Pacific. Data collected from stations located on the southern part of China and a polar station on an island located on the north Atlantic ridge-transform system also indicate large normalized transverse amplitudes. Small normalized transverse amplitudes are observed in mid-Eurasia and Australia, which are tectonically quiet regions on stable continents. In the American continents, large normalized transverse amplitudes are observed along the subduction zones and transform faults distributed along the western coast. The southern, central, and northern parts of North America, including eastern South America, also indicate 


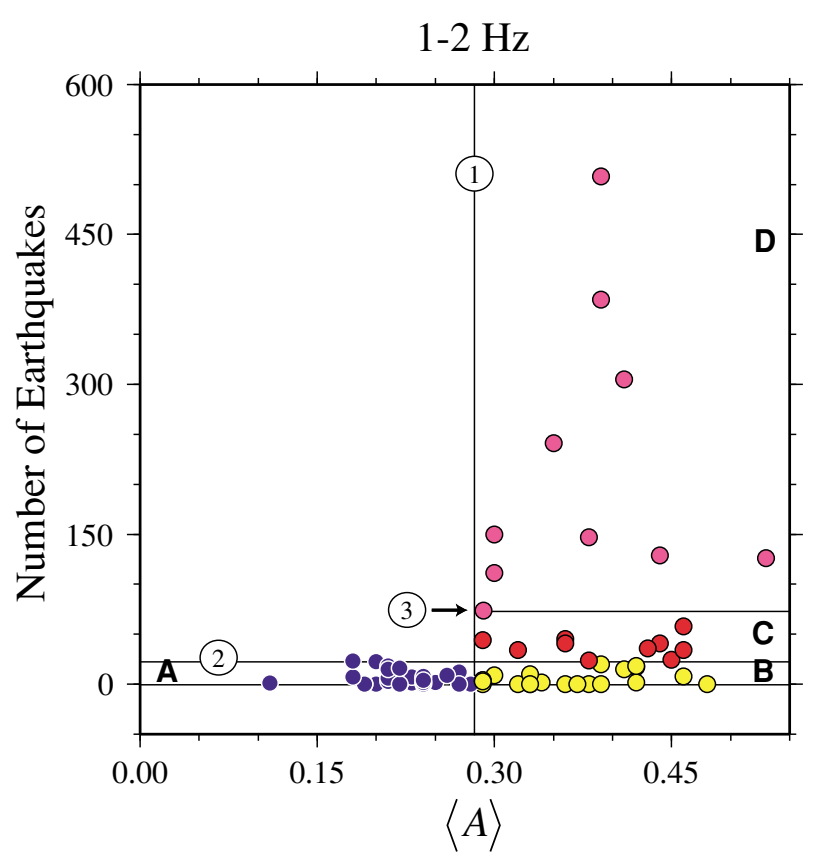

Fig. 2. Relation between normalized transverse amplitudes of 1-2 Hz frequency band and the number of earthquakes at each station. The number of earthquakes indicates those occurring within a $300-\mathrm{km}$ radius of each station during the period from 1973 to 2003. The encircled numbers represent the boundaries of categories A, B, C, and D (see text).

large normalized transverse amplitudes. Small amplitudes dominate in eastern North America. In the African continent, large normalized transverse amplitudes are found along the rift valley, while small normalized transverse amplitudes dominate on the rest of the continent, including Saudi Arabia. In Antarctica, large normalized transverse amplitudes are found at the South Pole station and in the western Hemisphere. Small normalized transverse amplitudes are located in the eastern Hemisphere.

\section{Relation to the Seismicity around the Stations}

We compared the normalized transverse amplitudes distribution in Fig. 1 with the seismicity of the past 30 years (1973-2003) of earthquakes with a magnitude larger than or equal to 5 in the PDE catalog provided by the NEIC at the USGS. We found that high seismicity regions are generally characterized by large normalized transverse amplitudes. It is plausible that a high seismicity reflects a deformation of the crust, high-stress accumulation-generating cracks, and faults, all of which must cause strong small-scale heterogeneity in the crust.

Since tectonically active regions can be characterized by current seismicity, we then compared the normalized transverse amplitudes with the number of earthquakes occurring within a 300-km radius of each station (Fig. 2). Stations located in high seismicity regions were found to always have large normalized transverse amplitudes. However, the data collected from stations in seismically inactive regions indicated not only small normalized transverse amplitudes but also large ones, implying that current seismicity cannot reflect all of the medium heterogeneity embedded in the lithosphere.
Based on these findings, we classify the lithosphere into four categories according to the observed normalized transverse amplitudes and the current seismicity. First, we divide the regions into two by setting a boundary in the middle of the minimum and maximum observed normalized transverse amplitude (boundary 1 in Fig. 2). Those regions having small normalized transverse amplitudes are denoted as category A. We then divide the other regions, all with large normalized transverse amplitudes, into three categories. We set boundary 2 at the position where the maximum number of earthquakes of 25 is observed in category A; category $\mathrm{B}$ is then characterized by a large normalized transverse amplitude with a seismicity as low as that of category A. Boundary 3 is set at the number of earthquakes of 75 , which is threefold larger than that of boundary 2 ; this divides the regions into categories $\mathrm{C}$ and $\mathrm{D}$. This classification is simple, but very useful for evaluating lithospheric heterogeneity. As these categories are denoted by different color scales in Fig. 1, it is clearly discernable that stations in category A are predominantly located on stable continents and that stations of categories $\mathrm{C}$ and $\mathrm{D}$ are distributed along collision zones and island arcs, respectively. This is consistent with results reported in previous studies, such as those of as Korn (1993) and Nishimura et al. (2002); that is, the heterogeneity of the lithosphere is correlated with the tectonic settings. The lateral variation in Q-values in the uppermost mantle (at a depth of less than $200 \mathrm{~km}$ ) determined by Gung and Romanowicz (2004) from surface waveform analyses is also consistent with our results. Since the normalized transverse amplitudes determined by applying a long (30 s) time window indicate spatial variations quite similar to the results obtained with a 20 -s time window, such a correlation with Q-values in the uppermost mantle implies that lateral heterogeneity extends from the crust to the deeper portions of the lithosphere.

However, large normalized transverse amplitudes at 17 stations of category B were observed in regions where very low seismicity is observed, including nine stations situated in regions where no earthquake of $M \geq 5$ has ever been observed (stations indicated by arrows in Fig. 1). The seismicity map of smaller earthquakes $(M \geq 3)$ detected by NEIC/USGS shows that a few earthquakes are distributed around four (ALE, HKT, XAN and TAU) of the nine stations; hence, large normalized transverse amplitudes at these stations may not be a manifestation of the heterogeneous structure of a completely inactive and stable lithosphere. Station MDJ is situated in close proximity to a Holocene volcano (Jingpohu) in eastern China; hence, the observed large normalized transverse amplitude can be considered to represent the medium heterogeneity in the lithosphere that is formed by volcanic processes. However, it is worth mentioning that no seismic and volcanic activities were recognized at four stations (BILL, FFC, BDFB, and SPA) located on the eastern Eurasian, central North American and central South American continents, and at the South Pole in Antarctic. The origin of the strong lateral heterogeneity existing beneath these stations is still unknown, but may be related with ancient activities of the lithosphere.

We also compared the spatial variation of the observed large normalized transverse amplitudes in category B with a 


\section{$1-2 \mathrm{~Hz}$}

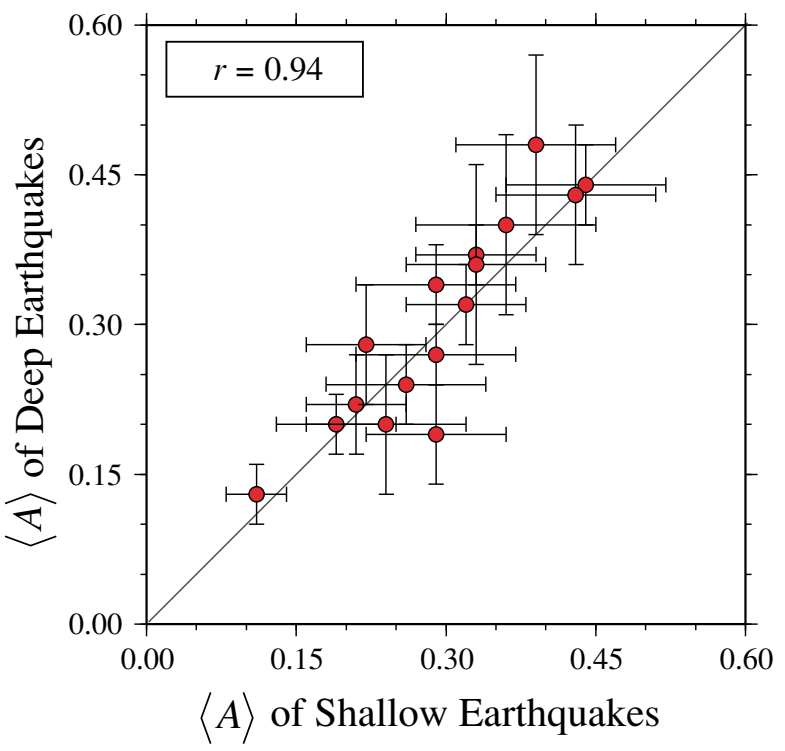

Fig. 3. Comparison of normalized transverse amplitudes estimated from deep earthquakes (depth $>300 \mathrm{~km}$, M: 5-7, period: 1987-2000) and shallow ones recorded at stations on the western Pacific. The results of 1-2 Hz frequency band are shown. Normalized transverse amplitudes of each station (circles) are plotted with standard deviations (bars).

global digital map of sediment thickness on a scale of $1 \times 1^{\circ}$ (available at http://mahi.ucsd.edu/Gabi/sediment.html; after G. Laske and G. Masters). Most of the stations were found to be located on a thin sediment layer with a thickness of less than $3 \mathrm{~km}$, and some of these stations on the thin sediment show large normalized transverse amplitudes, suggesting that the observed normalized transverse amplitudes reflect the heterogeneity of lithosphere. However, station HKT in the southern USA and station ENH in southeastern China are located on a thick sediment zone (more than $7 \mathrm{~km}$ thick). The large normalized transverse amplitudes observed at these stations are considered to be caused by a shallow sedimentary structure beneath the stations.

\section{Comparison of the Results from Shallow and Deep Earthquakes}

Normalized transverse amplitudes of teleseismic $P$ waves from shallow earthquakes are theoretically expected to be less affected by complex source time functions caused by depth phases, such as $p P$ and $s P$ phases as well as scattered waves originating around the focal zone, since the incident angle of the teleseismic $P$-wave to each station is limited to a narrow range directed towards the hypocenter. However, it would be much more useful to examine whether our results are affected to any great extent by the source itself and to assess just how much of the difference is caused from differences in focal depth difference. Figure 3 compares the normalized transverse amplitudes at stations in the western Pacific regions where both deep and shallow earthquakes are observed. The normalized transverse amplitudes from deep earthquakes $(>300 \mathrm{~km})$, which are less affected by heterogeneity around the focal area, are almost proportional to those estimated from shallow earthquakes. The correlation coefficient between them was estimated to be 0.94. Similar characteristics were observed in the frequency bands of $0.5-1 \mathrm{~Hz}$ and 2-4 Hz. Therefore, analyses of shallow earthquakes do enable us to extend the region which can be used to systematically characterize the medium heterogeneity beneath each station using teleseismic $P$-waves since shallow events are widely distributed on the globe and well recorded at many stations around the world.

\section{Summary}

Through a global characterization of lithospheric heterogeneity, we have shown that the spatial changes in normalized transverse amplitudes in short periods correlate well with various tectonic settings, such as collision zones, subduction zones, stable continents, among others. The direct comparison of observed normalized transverse amplitudes and the number of earthquakes within a $300-\mathrm{km}$ radius of each station for the past 30 years revealed that seismically active regions are always characterized by large normalized transverse amplitudes. However, in tectonically inactive regions where the seismicity is low, the data from several stations indicate large normalized transverse amplitudes. Our results suggest that analyses of teleseismic $P$-waves are useful for detecting a heterogeneity uncorrelated with tectonic settings evaluated from recent seismicity, which may be related with ancient activities of the lithosphere.

Acknowledgments. We thank the Data Management Center of IRIS for providing seismic data. Our sincere thanks are given to G. Poupinet for his extensive comments. Thoughtful reviews by B. Romanowicz and an anonymous reviewer helped us to improve the manuscript. M. K. is supported by MEXT under the Grant-inaid from Japanese Government scholarship. This study is partially supported by the 21st Century COE Program "EASTEC" of Tohoku University.

\section{References}

Fehler, M., M. Hoshiba, H. Sato, and K. Obara, Separation of scattering and intrinsic attenuation for the Kanto-Tokai region, Japan, using measurements of $S$-wave energy versus hypocentral distance, Geophys. J. Int., 108, 787-800, 1992.

Gung, Y. and B. Romanowicz, Q tomography of the upper mantle using three-component long-period waveforms, Geophys. J. Int., 157, 813830, 2004.

Korn, M., Determination of site-dependent scattering $\mathrm{Q}$ from $P$-wave coda analysis with an energy-flux model, Geophys. J. Int., 113, 54-72, 1993. Langston, C. A., Structure under Mount Rainier, Washington, inferred from teleseismic body waves, J. Geophys. Res., 84, 4749-4762, 1979.

Megnin, C. and B. Romanowicz, The 3D shear velocity structure of the mantle from the inversion of body, surface and higher mode waveforms, Geophys. J. Int., 143, 709-728, 2000.

Nishimura, T., K. Yoshimoto, T. Ohtaki, K. Kanjo, and I. Purwana, Spatial distribution of lateral heterogeneity in the upper mantle around the western Pacific region as inferred from analysis of transverse components of teleseismic P-coda, Geophys. Res. Lett., 29(23), 2137, 2002.

Sato, H., Synthesis of vector-wave envelopes in 3-D random elastic media characterized by a Gaussian autocorrelation function based on the Markov approximation: Plane wave case, J. Geophys. Res., 111, B06306, 2006 (doi: 10.1029/2005JB004036)

Sato, H. and M. C. Fehler, Seismic Wave Propagation and Scattering in the Heterogeneous Earth, 308 pp, Springer, New York, 1998. 